\title{
Atmospheric radiation environment analyses based-on CCD camera at various mountain altitudes and underground sites
}

\author{
Pierre Li Cavoli ${ }^{1,2, a}$, Guillaume Hubert ${ }^{1, \mathrm{~b}}$, and José Busto ${ }^{2, \mathrm{c}}$ \\ ${ }^{1}$ The French Aerospace Laboratory (ONERA), 31000 Toulouse, France \\ ${ }^{2}$ The Center for Particle Physics of Marseilles (CPPM), CNRS/IN2P3, 13288 Marseilles, France
}

\begin{abstract}
The purpose of this paper is to discriminate secondary atmospheric particles and identify muons by measuring the natural radiative environment in atmospheric and underground locations. A CCD camera has been used as a cosmic ray sensor. The Low Noise Underground Laboratory of Rustrel (LSBB, France) gives the access to a unique low-noise scientific environment deep enough to ensure the screening from the neutron and proton radiative components. Analyses of the charge levels in pixels of the CCD camera induced by radiation events and cartographies of the charge events versus the hit pixel are proposed.
\end{abstract}

\section{Background and context}

The Earth's atmosphere is constantly bombarded by cosmic rays which come from the Sun and the space. These cosmic rays are essentially relativistic protons (85\%), helium nuclei $(13 \%)$ and heavy nuclei $(2 \%)$ [1]. When the cosmic rays penetrate the atmosphere, they interact with its constituents such as nitrogen and oxygen atoms. These interactions generate cascades of secondary radiation of every kind called secondary particles [2]. Protons, neutrons, electrons, positrons, muons, pions and heavy nuclei are the main products [3]. Their intensity depends on the altitude, the geomagnetic latitude and the Sun's activity. Among the secondary particles which reach the earth surface, muons are the most abundant [4]. Historically the ionizing power of muons was not took into account because of their weak interaction with matter. Nevertheless with the current nanometrical scales of electronics devices, the muons ionization wake can no longer be neglected [5]. Radiations induced Single Event Effect (SEE) have been identified as a major reliability issue for commercial electronic systems $[3,6,7]$. These perturbations are the consequence of a current pulse generated by the impact of energetic particles in sensitive electrodes of the circuit. Soft errors are caused by cosmic rays and alpha particles induced by the natural alpha emitters from materials. As process node scales down, soft errors affect increasingly electronic systems including circuits from advanced CMOS technologies. Soft errors, which appear at the end of the 60's for space applications, constitute nowadays a major challenge not only for embedded systems of space, and avionic applications, but also for any system operating at ground level [8] for

\footnotetext{
a e-mail: pierre.li_cavoli@onera.fr

b e-mail: guillaume.hubert@onera.fr

c e-mail: busto@cppm.in2p3.fr
} 
which a fault may have critical consequences. The constant progresses in integrated circuits manufacturing technologies result in significant reductions of transistor dimensions. This makes very large-scale integration circuits more and more sensitive to perturbations. Thus a set of new error mechanisms appear. With such advanced technologies the track effect, namely the radial ionization profile, is expected to be critical multiple-cell upsets being the main contributor [9-11]. As feature sizes scales down, charge collection at multiple nodes (i.e., charge sharing) due to a single particle hit results from the decrease spacing dimensions between cells [12-15]. Then the sensitivity to SEE is expected to increase and recent studies have shown the occurrence of soft-errors due to protons $[16,17]$ and to muons [18] for advanced nano-scales electronics. In order to know the muon contribution in soft-errors, the first requirement is to ensure a solid understanding of the muon radiation environment. That is why through this paper we will present the feasibility of characterizing the atmospheric muon environment with a CCD camera. Then we will describe the analyses of the CCD camera measurements run in atmospheric sites and also in low noise environment in the underground facilities provided by the LSBB and the Modane Underground Laboratory (MUL).

\section{Camera characterization}

\subsection{Low-noise characterization}

The PIXIS 2048 CCD camera from the Princeton Instruments series is a 4.2 million-pixel $(2048 \times 2048)$ of $13.5 \mu \mathrm{m}^{2}$ full frame CCD with an imaging area of $7.64 \mathrm{~cm}^{2}$ and a depletion region of $4 \mu \mathrm{m}$. The imaging process of the observation of an object is based-on the shifting of the charge collected in each pixel of the CCD matrix. When a charged particle crosses the imaging area, a charge level is induced in each pixel which was hit along the particle track. The total charge deposit by the incident particle can be restored by summation of all fractional charges in each adjacent hit pixel [19]. A first measurement has been made in the Modane Underground Laboratory in order to characterize the intrinsic camera noise. Burried at a depth of 4800 m.w.e, only very few secondary particles from cosmic rays succeed in reaching the laboratory. A lead castle has been placed all around the camera for shielding it from remaning radioactivity and rare secondary particles. The integrated fluence $\phi_{\text {int }}$ is calculated as the sum of the detected particles divided by the CCD sensitive surface $S_{\text {pixel }}$ for four days of measurement (Eq. 1) and is displayed as a function of the exposition number (Fig. 1).

$$
\int_{1}^{N_{\text {expo }}} \phi_{\text {int }}=\frac{\sum_{i=1}^{N_{\text {expo }} \text { detected particles }(i)}}{S_{\text {pixel }}}
$$

with 2821 detected particles by $\mathrm{cm}^{2}$ for four days of measurement and $S_{\text {pixel }}=7.64 \mathrm{~cm}^{2}$. Each exposition records charge pixels during 50 seconds before be cleaning out. From the integrated fluence the mean flux is extracted and give access to the intrinsic noise as (Eq. 2):

$$
\bar{\Phi}_{\text {flux } C C D}=\frac{\int_{1}^{N_{\text {expo }}} \phi_{\text {int }} \cdot T_{\text {expo }}}{N_{\text {expo }}}
$$

with $\int_{1}^{N_{\text {expo }}} \phi_{\text {int }}=369$ particles.cm ${ }^{-2}, T_{50 s}=72$ (number of expositions for one hour of measurement), $N_{\text {expo }}=6912$ expositions (for four days of measurements). This intrinsic noise is equal to 3.8 particles. $\mathrm{cm}^{-2} \cdot \mathrm{h}^{-1}$ and is substracted for the next measurements. 


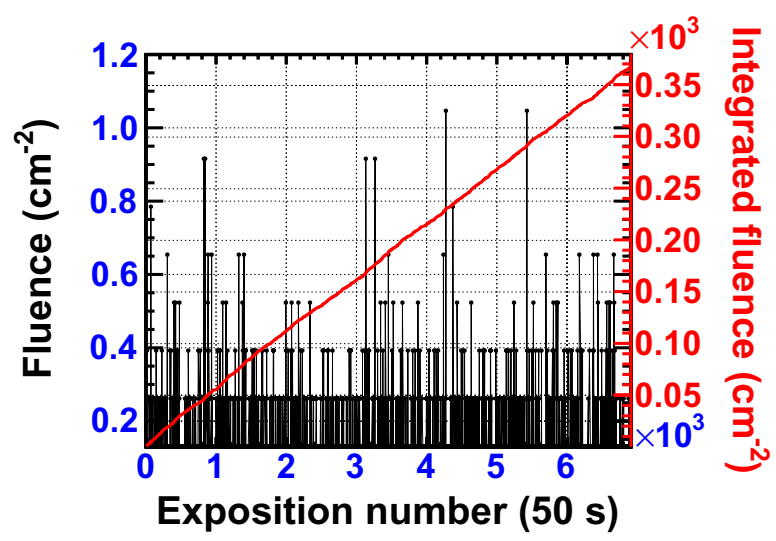

Figure 1. Fluence versus exposition number at the Modane Underground Laboratory.

\subsection{Radon and gamma irradiations}

In an underground location, particles emitted due to natural radiation can interact with the detector. Because the CCD camera detect all kind of ionizing particles and is not directly able to ascertain their nature, we have investigated the camera sensitivity to the products of the natural radioactivity as the radon and gamma. In laboratory, we have assembled a cuve fill of radon by an injecting pump with the CCD camera placed inside in a closed-circuit system. The time of the experiment has been limited to two hours in order to prevent the overheating of the camera. The detected particles flux is displayed with and without radon (Fig. 2a). A mean flux of 70 particles.cm ${ }^{-2} \cdot \mathrm{h}^{-1}$ is obtained with radon namely 25 particles. $\mathrm{cm}^{-2} \cdot \mathrm{h}^{-1}$ more than with the empty cuve. Thus the CCD camera is sensitive to the impact of radon. In the same manner, the camera has been placed through contact with a light radium source $\left({ }^{226} \mathrm{Ra}\right)$ which emit gamma, alpha and beta through the ${ }^{238} \mathrm{U}$ decay chain. The CCD camera is also sensitive to gamma rays by detecting 47 particles. $\mathrm{cm}^{-2} \cdot \mathrm{h}^{-1}$ more than with the reference measurement (Fig. 2b).

\section{Atmospheric and underground measurements}

\subsection{Detected particle flux analyses}

After the camera characterization we used the LSBB for atmospheric and underground sites. For the first kind of measurements, we had access to "Vestale" which is a mountain location at the summit of the "Grande Montagne" perched at $1067 \mathrm{~m}$ above the sea-level [20]. The detected particles flux obtained at "Vestale" is compared with the one obtained at the Center for Particle Physics of Marseilles (CPPM). Their mean flux is 161 particles. $\mathrm{cm}^{-2} \cdot \mathrm{h}^{-1}$ and 90 particles. $\mathrm{cm}^{-2} \cdot \mathrm{h}^{-1}$ respectively for an altitude of +1067 and $+150 \mathrm{~m}$ (Fig. 3). For the underground measurements, the LSBB has allowed us to use two rooms of different depth. The first one is buried at $60 \mathrm{~m}$ under the rocks at the laboratory entrance. The second one, which is called "Rameau" is the deepest part of the LSBB and is buried at a depth of $500 \mathrm{~m}$. A first measurement was made in 2011 (Fig. 4). The detected particle flux at $500 \mathrm{~m}$ is greater than the flux at $60 \mathrm{~m}$. This result can be explained by the presence of radon during the measurement. Indeed the radon radioactivity increases with the depth and with the difference between the temperature in the buried room and the temperature at the ground surface. When the temperature on the top of the mountain is greater than in the galleries of the LSBB, the 

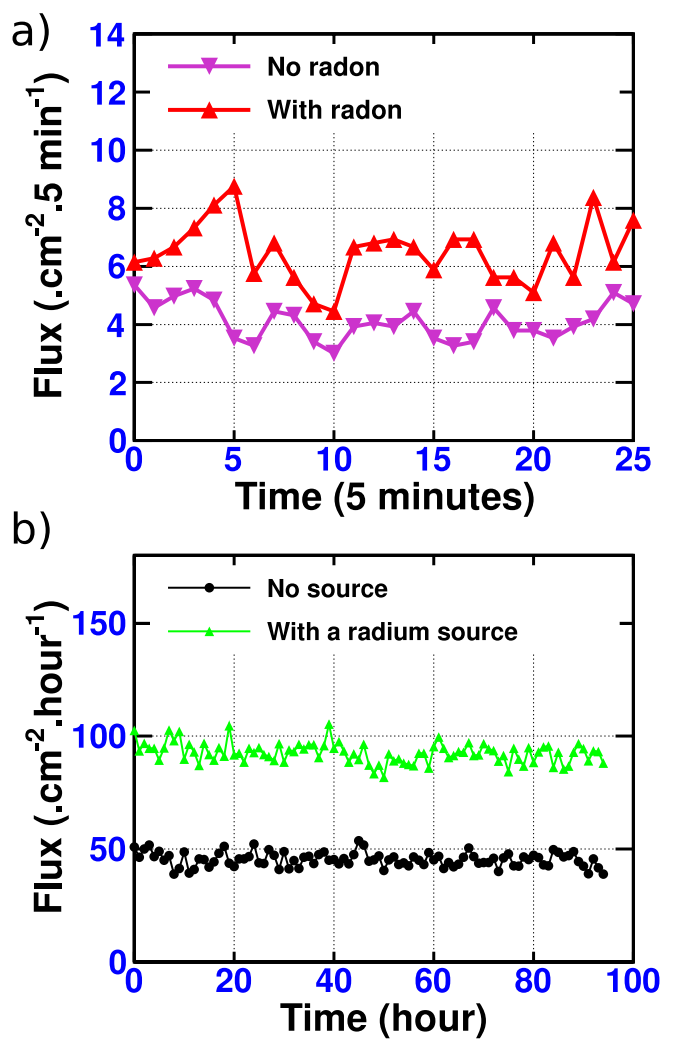

Figure 2. Detected particle flux: (a) in the presence of radon (b) with a radium source.

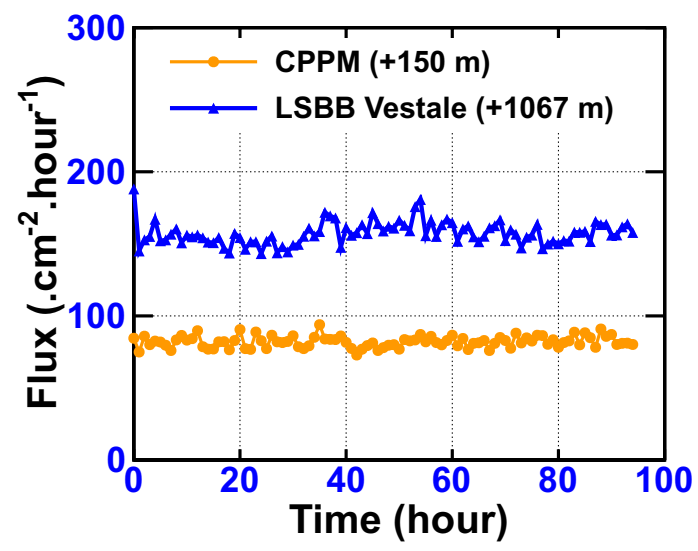

Figure 3. Detected particle flux for the atmospheric sites.

radon tends to be released from the rocks and go down toward the galleries. This situation occurs when the outside temperature reaches nearly $15^{\circ} \mathrm{C}$. Nevertheless, in 2012 a ventilation system was set up in order to reduce the radon flux. This system was efficient and allowed to decrease the flux of the detected particles by a factor of 2.4, as it is visible from the new measurement realized in 2015 (Fig. 4). 


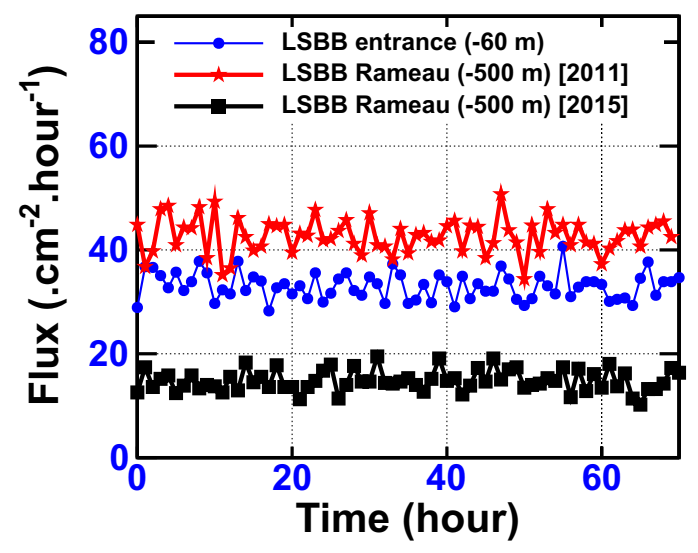

Figure 4. Comparison of the detected particle flux with (2015) and without (2011) ventilation system.

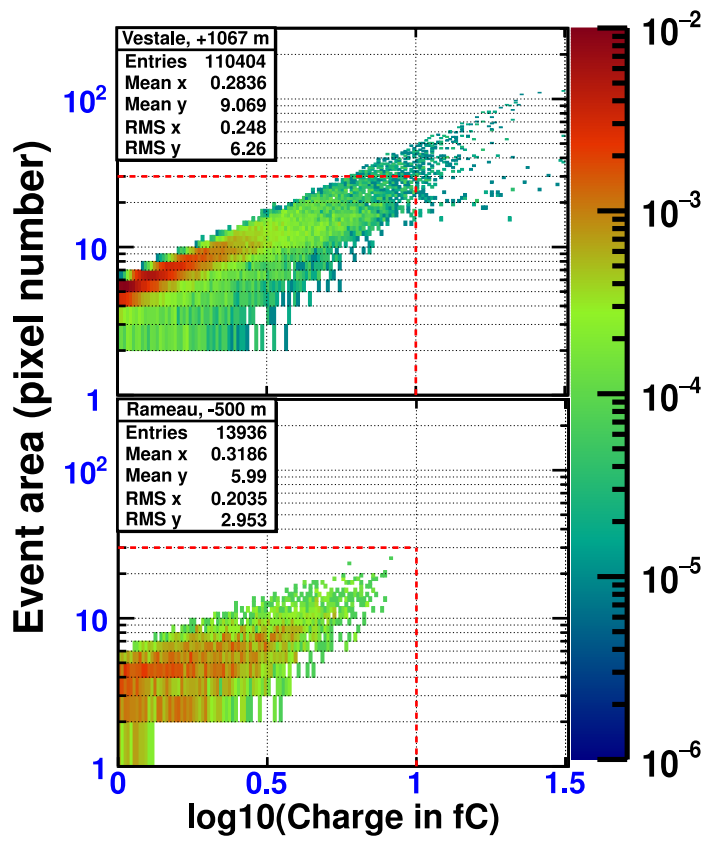

Figure 5. Event cartography: (a) atmospheric site $(+1067 \mathrm{~m})$ (b) underground site $(-500 \mathrm{~m})$.

\subsection{Event cartography analyses}

From these measurements the event cartography of "Vestale" and "Rameau" (respectively $+1067 \mathrm{~m}$ and $-500 \mathrm{~m}$ ) are compared (Fig. 5). The scale colors represents the occurence probability of the event. Both cartographies are normalized on four days. At $+1067 \mathrm{~m}$, 110404 events are detected whereas at $-500 \mathrm{~m}$ only 13936 events are. The red dashed lines (at $10 \mathrm{fC}$ and 30 hit pixels) represent the limited area detection by the underground measurement site: outside this area no events are detected at $-500 \mathrm{~m}$. This cut corresponds to the absorption of the hadronic component (mainly protons and neutrons) by the depth of rocks. Because muons are the only particles which can penetrate such a depth (except 
the neutrino that CCD camera can not detect), only muons and the irreductible natural radioactivity which can not be vanished remains at $-500 \mathrm{~m}$. The red core visible at $+1067 \mathrm{~m}$ located in a zone which spread from 4 to 10 hit pixel and from 1 to $3 \mathrm{fC}$, represents the most probable events and is also visible at $-500 \mathrm{~m}$ but it is more diffused. Because muons are the most abundant secondary particles at the ground surface [4], this core can be attributed to the muon charge deposit pattern.

\section{Conclusion}

Through this paper we have described charge level analyses from a CCD camera utilized as a cosmic ray sensor. Thanks to several different depths of rocks and a high-mountain altitude site, the LSBB appeared to provide a remarkable measurement site for investigating secondary particles from cosmic rays. Combined with measurements at the MUL, the analyses of the events observed by the CCD camera give access to the detected particles flux and the charge deposition pattern. Results showed that high charge level events seen on atmospheric sites can be considered as hadronic component (mainly neutrons and protons) while low charge levels and punctual events are induced by muons which are able to generate up to $3 \mathrm{fC}$ in the CCD camera. Hence, thanks to double level of measurement sites, muon discrimination from other secondary particles has been investigated.

\section{References}

[1] G. Reitz, Radiation Protection Dosimetry (1993).

[2] J.L. Barth, C. Dyer, E. Stassinopoulos, Nuclear Science, IEEE Transactions on 50, 466 (2003).

[3] J.F. Ziegler, IBM journal of research and development 40, 19 (1996).

[4] K. Nagamine, Introductory muon science (Cambridge University Press, 2003).

[5] B.D. Sierawski, Mendenhall et al., Nuclear Science, IEEE Transactions on 57, 3273 (2010).

[6] D. Lambert, J. Baggio, G. Hubert, V. Ferlet-Cavrois, O. Flament, F. Saigne, F. Wrobel, H. Duarte, J. Boch, B. Sagnes et al., Nuclear Science, IEEE Transactions on 52, 2332 (2005).

[7] D. Lambert, J. Baggio, G. Hubert, P. Paillet, S. Girard, V. Ferlet-Cavrois, O. Flament, F. Saigne, J. Boch, B. Sagnes et al., Nuclear Science, IEEE Transactions on 53, 1890 (2006).

[8] G. Hubert, R. Velazco, C. Federico, A. Cheminet, C. Silva-Cardenas, L. Caldas, F. Pancher, V. Lacoste, F. Palumbo, W. Mansour et al., Nuclear Science, IEEE Transactions on 60, 2418 (2013).

[9] A. Akkerman, J. Barak, D. Emfietzoglou, Nuclear Instruments and Methods in Physics Research Section B: Beam Interactions with Materials and Atoms 227, 319 (2005).

[10] R.K. Lawrence, A.T. Kelly, Nuclear Science, IEEE Transactions on 55, 3367 (2008).

[11] G. Hubert, P. Li Cavoli, C. Federico, L. Artola, J. Busto, IEEE Transactions on Nuclear Science 62, 2837 (2015).

[12] O.A. Amusan, A.F. Witulski, L.W. Massengill, B.L. Bhuva, P.R. Fleming, M.L. Alles, A.L. Sternberg, J.D. Black, R.D. Schrimpf, Nuclear Science, IEEE Transactions on 53, 3253 (2006).

[13] B.D. Olson, O.A. Amusan, S. Dasgupta, L.W. Massengill, A.F. Witulski, B.L. Bhuva, M.L. Alles, K.M. Warren, D.R. Ball, IEEE Transactions on Nuclear Science 4, 894 (2007). 
[14] A.M. Francis, D. Dimitrov, J. Kauppila, A. Sternberg, M. Alles, J. Holmes, H.A. Mantooth, Nuclear Science, IEEE Transactions on 56, 3109 (2009).

[15] J. Black, D. Ball, W. Robinson, D. Fleetwood, R. Schrimpf, R. Reed, D. Black, K. Warren, A. Tipton, P. Dodd et al. (2008).

[16] B.D. Sierawski, J.A. Pellish, R.A. Reed, R.D. Schrimpf, K.M. Warren, R.A. Weller, M.H. Mendenhall, J.D. Black, A.D. Tipton, M.A. Xapsos et al., Nuclear Science, IEEE Transactions on 56, 3085 (2009).

[17] G. Hubert, S. Duzellier, F. Bezerra, R. Ecoffet, in Radiation and Its Effects on Components and Systems (RADECS), 2009 European Conference on (IEEE, 2009), pp. 179-186.

[18] G. Hubert, L. Artola, D. Regis, Integration, the VLSI journal 50, 39 (2015).

[19] G. Hubert, A. Cheminet, T. Nuns, V. Lacoste, Nuclear Science, IEEE Transactions on (2013).

[20] G. Waysand, et al., NIMPR Section A (2000). 